\title{
DEVELOPMENT OF NEW DRUG FORMULATIONS: CETIRIZINE-POLYMERS NANOPARTICLES
}

\author{
SAMER HASAN HUSSEIN-AL-ALI ${ }^{1,2^{*}}$, MOHD ZOBIR HUSSEIN $^{3}$, RAMI AYOUB $^{1}$, \\ SHARIDA FAKURAZI ${ }^{4,5}$, QAIS IBRAHIM ABDALLAH ABUALASSAL ${ }^{1}$, \\ and YOUSEF AL-DALAHMEH ${ }^{1}$
}

${ }^{1}$ Faculty of Pharmacy, Isra University, P.O. Box 22, Amman 11622, Jordan

${ }^{2}$ Faculty of Science, Isra University, P.O. Box 22, Amman 11622, Jordan

${ }^{3}$ Materials Synthesis and Characterization Laboratory, Institute of Advanced Technology (ITMA),

Universiti Putra Malaysia, 43400UPM, Serdang, Selangor, Malaysia

${ }^{4}$ Laboratory of Vaccines and Immunotherapeutics, Institute of Bioscience, Universiti Putra Malaysia, 43400UPM, Serdang, Selangor, Malaysia

${ }^{5}$ Department of Human Anatomy, Faculty of Medicine and Health Sciences, Universiti Putra Malaysia, 43400UPM, Serdang, Selangor, Malaysia

\begin{abstract}
Cetrizine (Cet)-encapsulated chitosan- (CSNP), alginate- (AlgNP) and chitosan-alginate- nanoparticles (CSNP-AlgNP) were prepared using the ion-ion interaction method to form Cet-CSNPs, Cet-AlgNPs, Cet-CSNPs-AlgNPs nanoparticles, respectively. The three nanoparticles were then subjected to drug delivery studies. The nanoparticles were prepared by the dropwise addition of $\mathrm{Cet}$ or $\mathrm{CaCl}_{2}$ or TPP solution to Alg and CS solution, depending on the nanoparticles prepared. These Cet-CSNPs, Cet-AlgNPs, CetCSNPs-AlgNPs were around 96, 95, and $109 \mathrm{~nm}$ in size, respectively, with zeta potential values of +6.7 , -5.6 , and $-6.4 \mathrm{mV}$, respectively. The percentage loading efficiency was found to be 20,45 , and $35 \%$ for Cet-CSNPs, Cet-AlgNPs, and Cet-CSNPs-AlgNPs, respectively. Fourier transform-infrared spectroscopy and thermogravimetric analysis data indicate the successful formation of Cet-CSNPs, Cet-AlgNPs, CetCSNPs-AlgNPs. In vitro release of Cet shows about $83 \%, 90 \%$, and $75 \%$ release in $30 \mathrm{~h}$ for Cet-CSNPs, Cet-AlgNPs, and Cet-CSNPs-AlgNPs, respectively, and were best fitted into Hixson-Crowell, Pseudo-second, and Higuchi models for Cet-CSNPs, Cet-AlgNPs, Cet-CSNPs-AlgNPs, respectively. The in vitro cytotoxicity data for the three nanoparticles does not show any toxic effect toward 3T3 cell lines, at least up to $100 \mu \mathrm{g} / \mathrm{mL}$. The nanoparticles have good potential to be used as the extended-release formulation of Cet.
\end{abstract}

Keywords: cetirizine, chitosan nanoparticles, alginate nanoparticles, chitosan-Alginate nanoparticles, sustained release, 3T3 cell lines

One of the major research areas in the drug delivery system is the preparation of polymer nanoparticles that are capable to deliver drugs with high efficacy and smart targeted sites which prevent the spread of antibiotic resistance and improve patient compliance (1). Polymer nanoparticles can improve drug stability (2), improve the time of the therapeutic effect (3), and prevent or minimize drug degradation (4). There is a great interest in the application of nanoparticles for the synthesis of biodegradable polymers, natural biopolymers, lipids, and polysaccharides as biomaterials for the delivery of drugs.

Recently, the use of alginate and chitosan nanoparticles to deliver drugs has become of great interest. Both of them have been often used in the pharmaceutical industry for controlling the kinetics of the drug release (5).

Alginate (Alg) is a linear polysaccharide with alternating blocks of 1-4 linked $\alpha$-L-guluronic and $\beta$-D-mannuronic acid residues. Alg has the potential to be used for drug delivery and cell encapsulation. AlgNPs can be prepared easily by gelation with crosslinkers such as calcium ions (6).

Chitosan (CS) is a linear polysaccharide polymer with glucosamine and $\mathrm{N}$-acetyl glucosamine units. It is biocompatible, biodegradable, and nontoxic (7). CS contains three reactive functional groups; both primary and secondary hydroxyl groups at the $\mathrm{C}-2, \mathrm{C}-3$, and $\mathrm{C}-6$ positions, and also the amino group and acetamide group. CS has

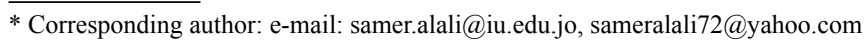


very good potential for various biomedical applications, such as for treatment of recalcitrant lesions of cutaneous leishmaniasis (8), antibacterial wound dressing (9), delivery of drugs (10-12), and antioxidant and metal chelating. Different drugs were loaded on chitosan nanoparticles; antibiotic drugs like ampicillin, doxycycline, metronidazole, and ceftriaxone, anticancer drugs such as 5-fluorouracil, saponin, letrozole, doxorubicin, and paclitaxel; Parkinson's disease drugs such as selegiline; central nervous system drugs such as thiocolchicoside and anti-hypertension drugs such as perindopril erbumine, etc.

The opposite charge network of CSNPs is formed by the interaction between the protonated amine functional groups $\left(\mathrm{NH}_{3}^{+}\right)$with an anionic phosphate group. In the case of AlgNPs, the anionic carboxyl group of alginate and cationic calcium ions reacted with each other. The polyelectrolyte network of CSNPs-AlgNPs is formed by the interaction between the dissociated anionic carboxyl groups of alginate with the protonated cationic amino group of chitosan. Intra- and inter-chain hydrogen bonding between different parts of the polysaccharides in CSNPs, AlgNPs, and CSNPS-AlgNPs structures will form (13). The formation of these nanoparticles depends on the order of mixing, the chemical composition of polymers, temperature, the flexibility of polymers, ionic strength, $\mathrm{pH}$, and reagent concentration (14).

Cetirizine dihydrochloride (Cet), 2-[2-[4-[(4chlorophenyl)-phenyl methyl] piperazin-1-yl] ethoxy] acetic acid dihydrochloride is one example of a second-generation antihistamine used to treat allergic rhinitis (hay fever), dermatitis, and urticaria (15). Different nanocarriers such as zinc-layered hydroxide nanoparticles (16) and carbon nanotubes (17) have been used, where Cet was used as the guest of these nanocarriers.

The quick disintegration of traditional, cetirizine tablets may result in primary "burst-released" kinetics, which may cause rapid drug absorption leading to high serum concentration with possible adverse effects such as local irritation to gastrointestinal (GI) mucous membranes (18-20). Disadvantages could be avoided by control the release rate of cetirizine dihydrochloride and cover the bitter taste, using design and develop a rational delivery system as modified chitosan nanocarriers $(20,21)$.

The purpose of the current study was to assess the possibility of CSNPs, AlgNPs, and CSNPsAlgNPs nanoparticles to be used as carriers for cetirizine. The challenge of this work was to encapsulate cetirizine drugs onto CSNPs, AlgNPs, and
CSNPs-AlgNPs, which were prepared by ionotropic gelation. Cet was selected as a model drug for the preparation of the formulations for better-controlled release properties.

\section{MATERIALS AND METHODS}

\section{Materials}

Cetirizine hydrochloric acid $\left(\mathrm{C}_{21} \mathrm{H}_{25} \mathrm{ClN}_{2} \mathrm{O}_{3} \cdot 2 \mathrm{HCl}\right.$, molecular weight: 461.81) was purchased from Sigma-Aldrich, at $99.79 \%$ purity. CS with low molecular weight (10-120 kDa, 90\% deacetylation, SigmaAldrich), low viscosity sodium alginate (10-100 kDa), and sodium tripolyphosphate (TPP), was purchased from Sigma Aldrich, at $98.0 \%$ purity.

\section{Cells and cytotoxicity reagent}

Normal mouse fibroblast cells (3T3) were obtained from the American Type Culture Collection (ATCC, Manassas, VA, USA). The MTT assay kit was obtained from Sigma-Aldrich (Steinheim, Germany).

\section{Preparation of the nanoparticles \\ Preparation of solutions}

CS solution was prepared by dissolving about $300 \mathrm{mg}$ of CS into $1.0 \%(\mathrm{v} / \mathrm{v})$ of acetic acid and the solution was then stirred at $500 \mathrm{rpm}$ for $3 \mathrm{~h}$, and heated to $30^{\circ} \mathrm{C}$ until a viscous solution of CS was obtained.

Sodium TPP solution was prepared by dissolving about $300 \mathrm{mg}$ of TPP into $10 \mathrm{~mL}$ distilled water.

Cet and sodium TPP solution was prepared by mixing $300 \mathrm{mg}$ of TPP with $60 \mathrm{mg}$ of Cet. Both of them were dissolved into $10 \mathrm{~mL}$ distilled water.

For Alg and CaCl2 solutions, about $300 \mathrm{mg}$ of Alg powder was dissolved into $100 \mathrm{~mL}$ distilled water with overnight stirring, where the $\mathrm{pH}$ of this solution was around 7.1. The $\mathrm{CaCl}_{2}$ solution was prepared by dissolving $335 \mathrm{mg}$ of $\mathrm{CaCl}_{2}$ into $100 \mathrm{~mL}$ of distilled water.

For CS and CaCl2 solutions, $150 \mathrm{mg}$ of $\mathrm{CS}$ were dissolved into $1.0 \%(\mathrm{v} / \mathrm{v})$ acetic acid for the CS solution. At the same time, the $\mathrm{CaCl}_{2}$ solution was prepared by dissolving $167 \mathrm{mg} \mathrm{\textrm {CaCl } _ { 2 }}$ into distilled water, giving a clear solution.

Finally, for the Alg and TPP solutions, $150 \mathrm{mg}$ of Alg with $150 \mathrm{mg}$ of TPP were dissolved into $100 \mathrm{~mL}$ distilled water, giving a clear solution.

\section{Preparation of CSNPs and Cet-CSNPS}

The CSNPs nanoparticles were prepared by adding the TPP solution into the CS solution and adjusting the $\mathrm{pH}$ of the $\mathrm{CS}$ solution to 5.4 using $\mathrm{NaOH}$. 
The prepared CSNPs were stirred for $18 \mathrm{~h}$ and the resulting nanoparticles were centrifuged at $15000 \mathrm{rpm}$ for $10 \mathrm{~min}$ and dried at $50^{\circ} \mathrm{C}$ for $24 \mathrm{~h}$. The Cet-CSNPs were prepared by adding a mixture of TPP and Cet to the CS solution followed by the procedure for the preparation of the CSNPs.

\section{Preparation of the AlgNPs and Cet-AlgNPs}

The AlgNPs were prepared by adding the solution of $\mathrm{Alg}$ to $\mathrm{CaCl}_{2}$. The reaction content was adjusted to $\mathrm{pH} 5.4$ using $\mathrm{NaOH}$ solution. Prepared AlgNPs were stirred for $18 \mathrm{~h}$ and the resulting nanoparticles were centrifuged at $15000 \mathrm{rpm}$ for $10 \mathrm{~min}$ and dried at $50^{\circ} \mathrm{C}$ for $24 \mathrm{~h}$.

The Cet-AlgNPs were prepared using $60 \mathrm{mg}$ of Cet and $335 \mathrm{mg}$ of $\mathrm{CaCl}_{2}$. The mixture solution of Cet and $\mathrm{CaCl}_{2}$ was added to the $\mathrm{Alg}$ solution. The $\mathrm{pH}$ of the three components was increased from 4.5 to 5.4 using $\mathrm{NaOH}$ solution. The resulting Cet-AlgNPs were collected by centrifugation at $15000 \mathrm{rpm}$ for 10 minutes and dried at $50^{\circ} \mathrm{C}$ for $24 \mathrm{~h}$.

\section{Preparation of the CSNPs-AlgNPs and Cet-CSNPs-AlgNPs}

The CSNPs-AlgNPs were prepared by a method briefly describe as follows: the homogeneous mixture solution of Alg-TPP was dropped slowly into the $\mathrm{CS}-\mathrm{CaCl}_{2}$ solution for around $3 \mathrm{~h}$ while stirring at a high speed. The $\mathrm{pH}$ of the resulting solution was increased to $\mathrm{pH} 5.4$ using $\mathrm{NaOH}$ solution and the stirring was continued overnight. The CSNPs-AlgNPs formed were centrifuged at $15000 \mathrm{rpm}$ for $10 \mathrm{~min}$ and dried at $50^{\circ} \mathrm{C}$ for $24 \mathrm{~h}$. The Cet-CSNPs-AlgNPs were prepared as follows: the solution of Alg-TPP and Cet was dropped slowly into the $\mathrm{CS}-\mathrm{CaCl}_{2}$ solution for around $3 \mathrm{~h}$ while stirring at a high speed. The $\mathrm{pH}$ of the resulting solution was increased to $\mathrm{pH}$ 5.4 using $\mathrm{NaOH}$ solution and the stirring was continued overnight. The Cet-CSNPs-AlgNPs formed were centrifuged at $15000 \mathrm{rpm}$ for 10 minutes and dried at $50^{\circ} \mathrm{C}$ for $24 \mathrm{~h}$.

\section{The release of Cet from its nanoparticles}

Cet release profiles from the Cet-CSNPs, Cet-AlgNPs, and Cet-CSNPs-AlgNPs were determined using a phosphate buffer solution (PBS) of $0.01 \mathrm{~mole} / \mathrm{L}$ at $\mathrm{pH} 7.4$. About $100 \mathrm{mg}$ of the CetCSNPs, Cet-AlgNPs, and Cet-CSNPs-AlgNPs were added separately into $500 \mathrm{~mL}$ of the PBS media. The cumulative amount of Cet released into the PBS solution was measured at preset time intervals using a UV-Vis spectrophotometer at $231 \mathrm{~nm}$. The release medium $(2 \mathrm{~mL})$ was removed for the UV-vis analysis at given time intervals and it was then replaced with2 $\mathrm{mL}$ of the buffered solution.

To compare the release behavior of Cet from the nanoparticles with that from a physical mixture (prepared for this purpose), $10.0 \mathrm{mg}$ of the physical mixture of Cet $(5.0 \mathrm{mg})$ and nanoparticles $(50 \mathrm{mg})$ were used and placed into a cuvette and subjected to the UV-Vis spectrophotometer measurement.

The mean dissolution time (MDT) as a dissolution parameter was calculated for different formulation types according to Equation (1),

$$
\text { MDT }_{\text {in-vitro }}=\frac{\sum_{\mathrm{i}=1}^{\mathrm{n}} t_{\text {mid }} \Delta M}{\sum_{\mathrm{i}=1}^{\mathrm{n}} \Delta M}
$$

where $I$ is the dissolution sample number, $\mathrm{n}$ is the number of dissolution times, $\mathrm{t}$ is the time at the midpoint between times $t_{i}$ and $t_{i-1}, M$ is the amount of drug dissolved between time $t_{i}$ and $t_{i-1}$.

\section{The loading efficiency of Cet}

The loading efficiency of Cet in various nanoparticles was determined using Equation (2) as given below. The amount of free Cet in the supernatant was collected by a centrifuge at $15000 \mathrm{rpm}$ and measured by a UV-vis spectrophotometer. The Cet loading efficiency in the nanoparticles was calculated as follows:

$$
=\frac{\begin{array}{c}
\text { Loading Effciency } \%= \\
\text { mass of nanoparticles }
\end{array} \times 100}{\mathrm{C}_{\mathrm{t}}-\mathrm{C}_{\mathrm{f}}}
$$

where $C_{t}$ is the initial mass of the drug used in the experiment and $\mathrm{C}_{\mathrm{f}}$ is the mass of Cet in the supernatant, therefore $\left(\mathrm{C}_{\mathrm{t}}-\mathrm{C}_{\mathrm{f}}\right)$ is the mass of Cet which binds with the formulation.

The procedure which was used to determine the $\mathrm{C}_{\mathrm{f}}$ is as follows,

(i.) centrifuge the nanoparticles at $15000 \mathrm{rpm}$ for $10 \mathrm{~min}$ and collect the remaining unloaded Cet in the supernatant

(ii.) measure the absorbance for the supernatant and calculate the mass of Cet in the supernatant using a calibration curve.

After centrifuging, washing, and drying, the collected mass of the final sample was kept in a sample bottle and the loading efficiency of the drug was calculated according to Equation 1. 


\section{The effect of $\mathrm{pH}$ on the Zeta potential of the nanoparticles}

$\mathrm{pH}$ is the most important factor that affects the Zeta potential of the nanoparticles. To confirm these changes, the $\mathrm{pH}$ of titration was done using the dynamic light scattering (DLS) (Malvern Zetasizer Nano ZS) and the change in $\mathrm{pH}$ was plotted as a function of zeta potential.

\section{Cell culture and MTT cytotoxicity assays $3 T 3$ cells}

The 3T3 cells were obtained from Swiss albino mice (primary mouse embryonic fibroblast cells). The '3T3' designation refers to the abbreviation of "3-day transfer, inoculum $3 \times 10^{5}$ cells".

\section{Culture media}

The $3 \mathrm{~T} 3$ cells were maintained at $37^{\circ} \mathrm{C}$ and $5 \%$ $\mathrm{CO}_{2}$ in a humidified incubator with a cultured medium which consisted of five different components: Dulbecco's Modified Eagles Medium (DMEM) appended with $10 \%(\mathrm{v} / \mathrm{v})$ fetal bovine serum (FBS), $2 \mathrm{mM}$ L-glutamine, $100 \mathrm{unit} / \mathrm{mL}$ Penicillin and $100 \mathrm{microgram} / \mathrm{mL}$ streptomycin. After two days, the cells had grown to approximately $90 \%$ confluence. Then, they were detached with trypsin and seeded into a 96 -well plate at $1 \times 10^{5}$ cells $/ \mathrm{mL}$ and kept overnight for cell attachment.

After two days, the medium of each well was removed and $100 \mu \mathrm{g} / \mathrm{mL}$ of the prepared samples suspensions (pure Cet, CSNPs, AlgNPs, CSNPs-AlgNPs, Cet-CSNPs, Cet-AlgNPs, and Cet-CSNPs-AlgNPs) in varying concentrations in the cell culture medium were added to the wells $(0.0781,1.562,3.124,6.25,12.5$, 25,50 and $100 \mu \mathrm{g} / \mathrm{mL}$ ). The $3 \mathrm{~T} 3$ cells were cultivated for three more days after which the viability of the cells was determined by the (3-(4,5-dimethylthiazol2-yl)-2,5-diphenyl-tetrazolium-bromide) assay (22).

\section{Cytotoxicity test using the MTT assay}

The viability of the 3T3 cells was analyzed by the MTT assay. It is a colorimetric assay based on the reaction of the yellow tetrazole by mitochondrial dehydrogenase enzymes and leads to an insoluble purple formazan product (23). Firstly, the medium was removed from each well. Secondly, $100 \mathrm{~mL}$ of fresh medium and $10 \mathrm{~mL}$ of MTT solution $(5 \mathrm{mg} / \mathrm{mL}$ PBS) were added to each well and incubated for $4 \mathrm{~h}$ at $37^{\circ} \mathrm{C} / 5 \% \mathrm{CO}_{2}$. Two hours later, the dimethyl sulfoxide was added to the cells as a detergent reagent to stop the conversion and solubilize the formazan. The amount of purple product is directly correlated with the number of viable cells. The absorbance of the purple color that is formed is taken at a wavelength of $570 \mathrm{~nm}$ using a multiwell microplate reader. The experiment was performed in triplicates, and the result was expressed as the mean \pm SD. The cell viability calculated according to equation 3 .

$$
\begin{gathered}
\text { Cell Viability } \%= \\
=\frac{\text { Average of treated }}{\text { Average Control }} \times 100
\end{gathered}
$$

\section{Characterization of the nanoparticles Powder X-ray diffraction}

The entity and crystallinity of the nanoparticles were evaluated by the XRD diffraction technique. XRD patterns were taken with an XRD-6000 model diffractometer (Shimadzu, Tokyo, Japan) using the $\mathrm{CuK}_{\alpha}$ radiation $(\lambda=1.5406 \AA)$, at a high accelerating voltage of $40 \mathrm{kV}$ and $30 \mathrm{~mA}$ with $2 \theta=2-70$ degrees.

\section{Fourier transform infrared analysis}

To confirm the presence of chemicals or functional groups that constitute the nanoparticles, a Fourier-transform infrared (FTIR) analysis was done. The FTIR spectra were recorded over the range of $400-4000 \mathrm{~cm}^{-1}$ on a Perkin Elmer spectrometer (model Smart UAIR-two) with $4 \mathrm{~cm}^{-1}$ resolutions.

\section{Measurement of the Zeta potential}

The measurements of the zeta potential and particle size distribution of the nanoparticles were performed using a Zetasizer Nano-ZS (Malvern Instruments, Worcestershire, UK) at $25^{\circ} \mathrm{C}$.

\section{Transmission electron microscopy}

The particle shape, internal structure, and size of the nanoparticles were examined using transmission electron microscopy (TEM), (Hitachi H-7100, Tokyo, Japan) with an accelerating voltage of 80 and $200 \mathrm{kV}$.

\section{Thermogravimetric analysis}

The thermogravimetric analysis (TGA) was carried out using a Metter-Toledo 851e instrument (Switzerland) with a heating rate of $10^{\circ} \mathrm{C} \mathrm{min}{ }^{-1}$, in $150 \mu \mathrm{L}$ alumina crucibles at $30-900^{\circ} \mathrm{C}$.

\section{RESULTS AND DISCUSSION}

\section{$X$-ray diffraction}

Powder XRD patterns of Cet, CSNPs, CetCSNPs, AlgNPs, Cet-AlgNPs, CSNPs-AlgNPs and Cet-CSNPs-AlgNPs are presented in Figure 1. Free Cet (Figure 1A) shows crystalline properties due to the presence of sharp reflection peaks at $2 \theta=7.8^{\circ}$, $14.4^{\circ}, 18.2^{\circ}, 21.0^{\circ}, 24.2^{\circ}, 28.2^{\circ}, 30.8^{\circ}$ and $33.0^{\circ}(24)$. 
The XRD patterns of CSNPs (Figure 1B) appeared at $2 \theta=25.2^{\circ}$ and $49.7^{\circ}$ with amorphous patterns, which is due to the cross-linkages of CSNPs with TPP (25). The Cet-CSNPs (Figure 1C) show a weak peak at $2 \theta=25.2^{\circ}$ and $50.4^{\circ}$, with amorphous characteristics. This amorphous phase of the weak peak can be related to the loading of the Cet into CSNPs nanoparticles (26).

Figure 1D shows the XRD patterns of AlgNPs. The nanoparticles exhibited two characteristic reflection peaks at $2 \theta=8.6^{\circ}$ and $42.4^{\circ}$ with amorphous characteristics. Similar to AlgNPs, Cet-AlgNPs also show two characteristic reflection peaks at $2 \theta=10.2^{\circ}$ and $40.0^{\circ}$ with amorphous properties due to the presence of Cet in the Alg polymer (27). In addition, Figure 1E (Cet-AlgNPs) shows the presence of trace-free Cet, which is indicated by the $(\diamond)$ symbol at $2 \theta=16.2^{\circ}$.

XRD patterns of CSNPs-AlgNPs and CetCSNPs-AlgNPs are illustrated in Figures $1 \mathrm{~F}$ and $1 \mathrm{G}$, respectively. The XRD patterns of CSNPs-AlgNPs shows very low-intensity peaks at $2 \theta=24.4^{\circ}, 39.2^{\circ}$, and $45.2^{\circ}$, revealing the amorphous nature of the polymers. The Cet-CSNPs-AlgNPs (Figure 1G) shows a weak peak at $2 \theta=24.6^{\circ}, 38.6^{\circ}$, and $44.2^{\circ}$, with amorphous characteristics. The characteristic peaks of the Cet were absent in Cet-CSNPs-AlgNPs. This indicates that Cet was dispersed into both CS and Alg polymeric nanoparticles and there could be less or no free Cet in its crystalline form on the surface of the CSNPs-AlgNPs.

\section{Infrared spectroscopy}

Figure 2 shows the FTIR spectra and their assignment, respectively for Cet, Cet-CSNPs, CetAlgNPs, and Cet-CSNPs-AlgNPs. The FTIR spectra of Cet (Figure 2A) show broadband at $3432 \mathrm{~cm}^{-1}$, corresponding to the stretching of the hydroxyl groups of the $-\mathrm{COOH}$. A band at $3046 \mathrm{~cm}^{-1}$ is due to $\mathrm{C}-\mathrm{H}$ symmetry and asymmetry stretching in phenyl and chlorophenyl groups. A band at $1436 \mathrm{~cm}^{-1}$ was assigned to the $\mathrm{CO}$ bending, $\mathrm{C}-\mathrm{C}$ stretching, and $\mathrm{C}-\mathrm{H}$ wagging at piperazinyl ethoxy acetic acid. A band at $1187 \mathrm{~cm}^{-1}$ is suggested due to the presence of C-O-H bending and $\mathrm{C}-\mathrm{O}$ stretching vibration at piperazinyl ethoxy acetic acid groups. A band at $1093 \mathrm{~cm}^{-1}$ shows the $\mathrm{C}-\mathrm{H}$ rocking in the phenyl group and chlorophenyl group, as well as the $\mathrm{C}-\mathrm{Cl}$ stretching group. A band at $847 \mathrm{~cm}^{-1}$ was assigned to the $\mathrm{C}-\mathrm{C}$ stretching and $\mathrm{C}-\mathrm{O}$ stretching at piperazinyl ethoxy acetic acid and due to the C-N stretching (28).

The assignments of the FTIR spectra of CSNPs and Cet-CSNPs. A band at $3447 \mathrm{~cm}^{-1}$ is due to the stretching vibrations of the $\mathrm{O}-\mathrm{H}$ group. The presence of the absorption band at $1640 \mathrm{~cm}^{-1}$ for CSNPs

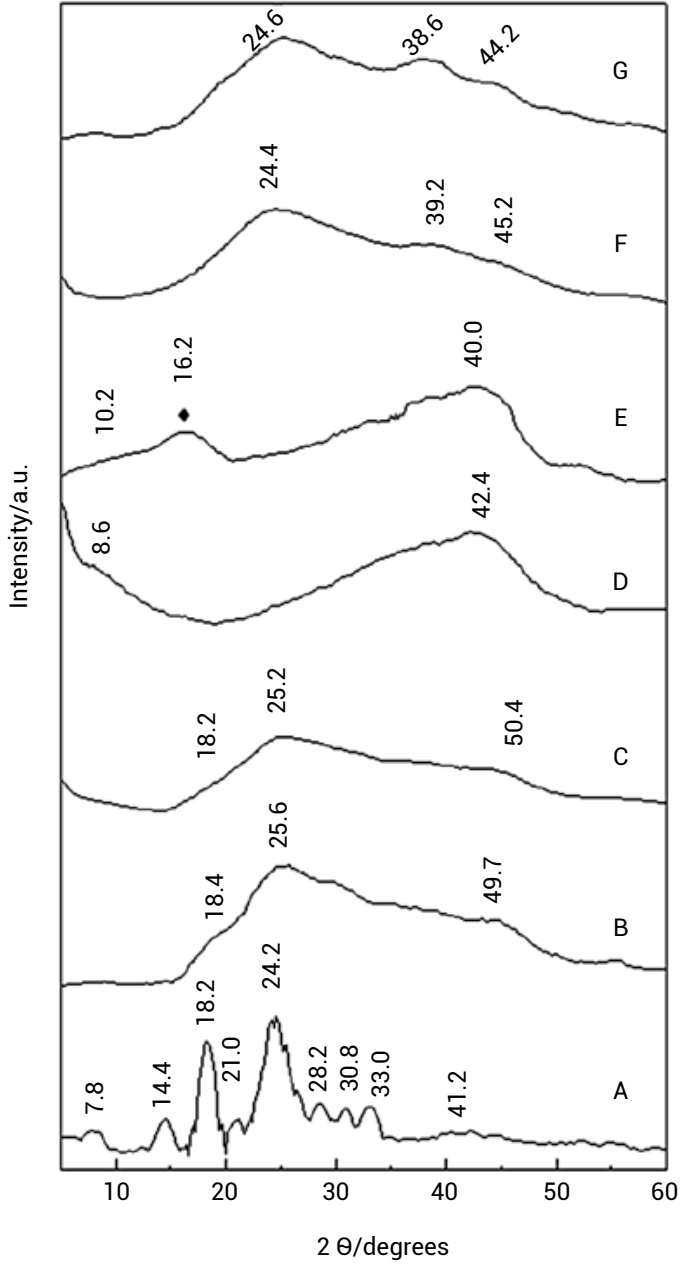

Figure 1. Powder X-ray diffraction patterns of Cet (A), CSNPs (B), Cet-CSNPs (C) AlgNPs (D) Cet-AlgNPs (E), CSNPs-AlgNPs (F) and Cet-CSNPs-AlgNPs (G).

and $1635 \mathrm{~cm}^{-1}$ for Cet-CSNPs is due to the protonated $\mathrm{NH}_{2}$ groups $\left(\mathrm{NH}_{3}^{+}\right)$. An absorption band at $1548 \mathrm{~cm}^{-1}$ for CSNPs and $1547 \mathrm{~cm}^{-1}$ for Cet-CSNPs is due to the $\mathrm{C}-\mathrm{H}$ bending vibration of the alkyl group. The $\mathrm{P}=\mathrm{O}$ group for TPP was observed at $1093 \mathrm{~cm}^{-1}$ for CSNPs and $1100 \mathrm{~cm}^{-1}$ for Cet-CSNPs (29).

The presence of Cet in Cet-CSNPs can be derived from two factors; first, the shift in some bands of Cet-CSNPs compared to CSNPs; the shift from 2948 to 2913,1640 to 1635 , and from 895 to $883 \mathrm{~cm}^{-1}$. This indicates that there exists an interaction between CSNPs and Cet. Secondly, some of the bands appeared in Cet-CSNPs and Cet FTIR spectra but were not observed in the FTIR spectrum of CSNPs. For example, a band at $1436 \mathrm{~cm}^{-1}$ which is due to the $\mathrm{C}-\mathrm{O}$ bending, $\mathrm{C}-\mathrm{C}$ stretching, and $\mathrm{C}-\mathrm{H}$ wagging at piperazinyl ethoxy acetic acid, and another band at $847 \mathrm{~cm}^{-1}$ which is assigned to $\mathrm{C}-\mathrm{C}$ stretching, and $\mathrm{C}-\mathrm{O}$ stretching at piperazinyl ethoxy acetic acid, and C-N stretching. 


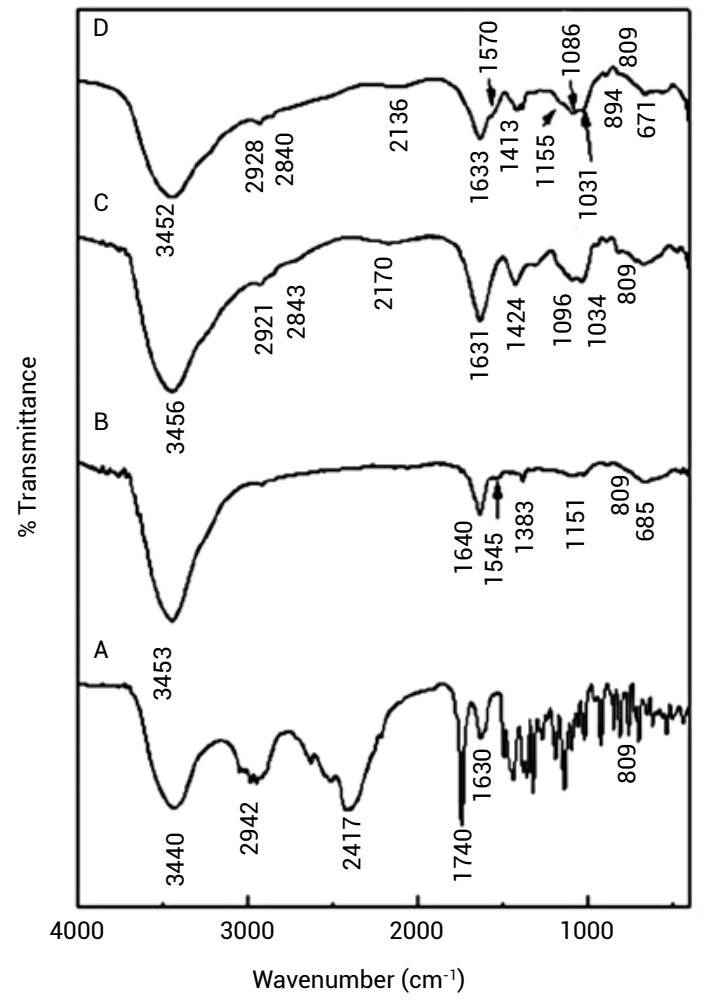

Figure 2. FTIR spectrum of the Cet (A), Cet-CSNPs (B), Cet-AlgNPs (C) and Cet-CSNPs-AlgNPs (D).

The absorption bands of AlgNPs show important bands for carboxylic, ether, and hydroxyl functional groups. The stretching vibration of the $\mathrm{O}-\mathrm{H}$ bond is observed at $3446 \mathrm{~cm}^{-1}$, while the stretching vibration of the aliphatic $\mathrm{C}-\mathrm{H}$ bond is observed at $2930 \mathrm{~cm}^{-1}$. Bands at 1631 and $1425 \mathrm{~cm}^{-1}$ were attributed to asymmetric and symmetric stretching vibrations of COO- of the salt ion, respectively. A band at $1108 \mathrm{~cm}^{-1}$ is attributed to the $\mathrm{C}-\mathrm{O}$ stretching vibration of pyranosyl ring (30).

The hydroxyl group $(\mathrm{OH})$ present in Cet-AlgNPs (Figure 2C) exhibits broadband at around $3446 \mathrm{~cm}^{-1}$. A band at $2921 \mathrm{~cm}^{-1}$ is due to the $-\mathrm{CH}_{2}$ group. A band at around $1034 \mathrm{~cm}^{-1}$ is attributed to the C-O-C group of the saccharide structure. In addition, bands at $1631 \mathrm{~cm}^{-1}$ and $1424 \mathrm{~cm}^{-1}$ are due to asymmetric and symmetric stretching of carboxylate salt groups (COO-) for both Cet anion and Alg.

The FTIR spectrum of the Cet-CSNPs-AlgNPs (Figure 2D) revealed the presence of characteristic bands of CSNPs and AlgNPs. This is because, during the preparation, we used TPP as a reagent for CS as well as the $\mathrm{CaCl}_{2}$ as a precipitating reagent for Alg. This also can be seen from the XRD results. In the FTIR spectra of mixed CSNPs and AlgNPs, as shown in Figure 2D, the Cet-CSNPs-AlgNPs has a narrower, more intense band at about $3446 \mathrm{~cm}^{-1}$, which is due to the forming of new hydrogen bonds between the functional groups in CSNPs and the functional groups of AlgNPs (31). In addition, the characteristic absorption band of Cet that appeared in the Cet-CSNPs-AlgNPs spectra indicates that Cet was encapsulated in the CS and Alg polymeric system.

\section{The interaction between Cet and the nanocarriers}

As indicated in the literature, CSNPs have been prepared by several methods such as ionotropic gelation methods, spray drying, emulsion cross-linking, template polymerization, and precipitation techniques (31). The ionic gelation method was adopted in this work, where gelation depends on the ionic

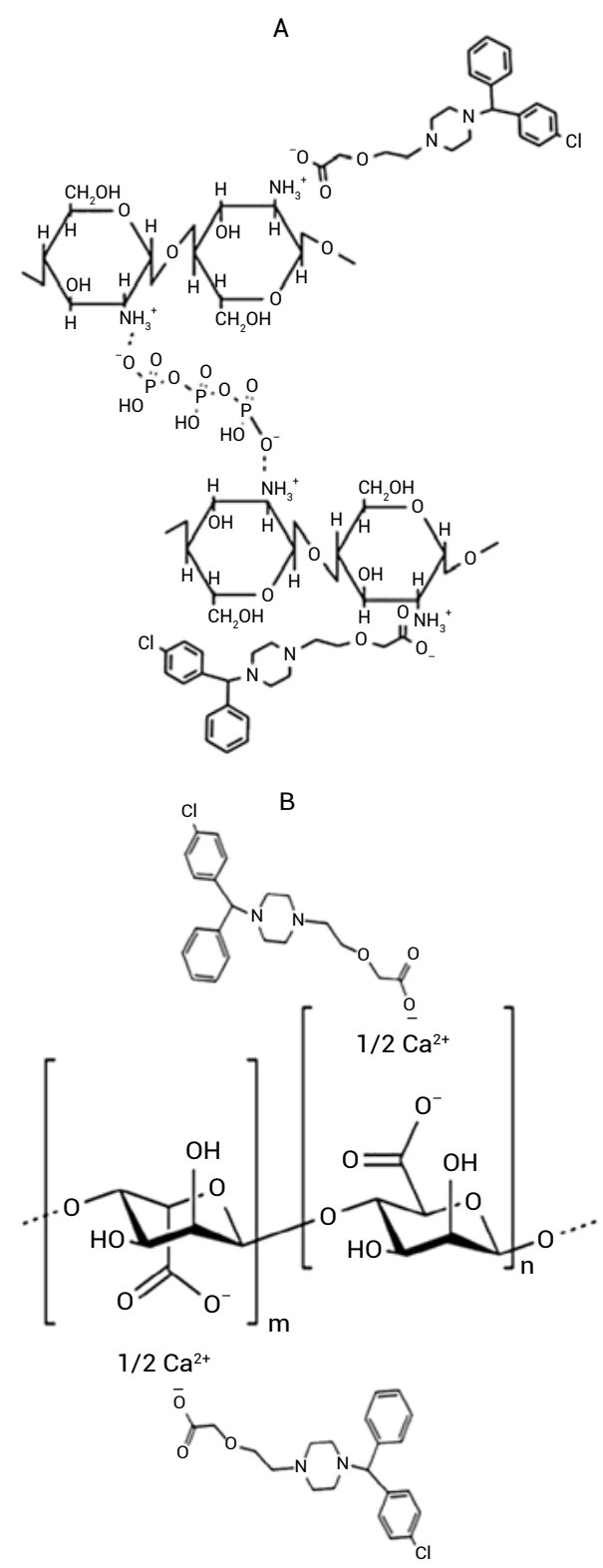

Figure 3. A schematic model showing plausible interaction between Cet and CSNPs (A), and Cet and AlgNPs (B). 
interaction between the amino groups $\left(\mathrm{NH}_{3}^{+}\right)$of $\mathrm{CS}$ which is positively charged, and TPP which is negatively charged (32). As shown in Figure 3A, one of the $\mathrm{NH}_{3}^{+}$groups react with TPP and the other one reacted with the negatively charged group of Cet.

During the formation of CetAlgNPs, the $\mathrm{Ca}^{2+}$ ions have two binding sites. The first binding site will interact with the Alg molecule and the second binding site will interact with Cet (Figure 3B).

\section{Transmission Electron Microscopy}

A typical TEM micrograph of the CSNPs, Cet-CSNPs, AlgNPs, CetAlgNPs, CSNPs-AlgNPs, and CetCSNPs-AlgNPs is shown in Figure 4A, B, C, D, and E, respectively. In this study, TEM images show physical aggregation of the CSNPs, AlgNPs, CSNPs-AlgNPs (Figure 4A, C, and E), and Cet-CSNPs, Cet-AlgNPs, and Cet-CSNPs-AlgNPs (Figure 4B, D, and F). As shown in Figures $3 \mathrm{~A}$ and $B$, the nanoparticles were seen to be spherical, distinct, and regular. The mean particle size diameter of the CSNPs was found to be $63 \mathrm{~nm}$, compared to 96 for Cet-CSNPs, similar to the one reported in the literature (33). The mean particle size of AlgNPs was found to be $112 \mathrm{~nm}$ (Figure 4C) which is slightly larger than that of Cet-AlgNPs, 94 nm (Figure 4D). The TEM image was found to be similar to the one in the literature (34).

The mean particle diameter measured from the TEM images for CSNPs-AlgNPs and Cet-CSNPsAlgNPs is shown in Figures $4 \mathrm{E}$ and $\mathrm{F}$, respectively. The Cet-CSNPs-AlgNPs were found to be smaller than CSNPsAlgNPs. The mean size for the CSNPsAlgNPs is around $121 \mathrm{~nm}$ with a round shape (Figure 4E) while the mean size for Cet-CSNPs-AlgNPs was found to be $109 \mathrm{~nm}$, which is similar to the one reported in the literature (35).

Figure 4. TEM images of CSNPs (A), Cet-CSNPs (B), AlgNPs (C), Cet-AlgNPs (D), CSNPs-AlgNPs (E) and Cet-CSNPs-AlgNPs (F).
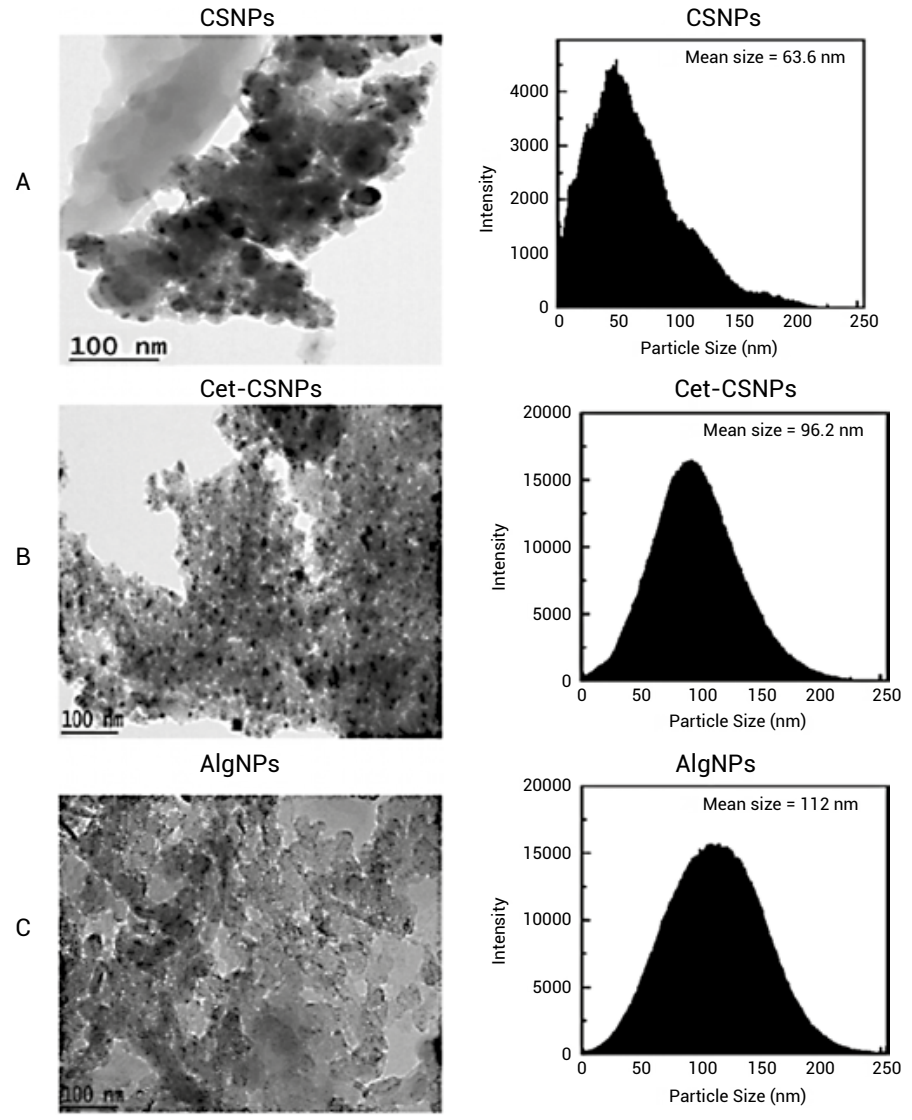

Cet-AlgNPs

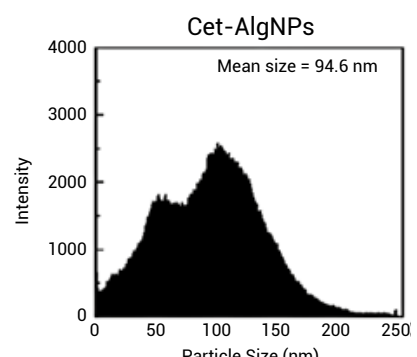

$\underline{100 \mathrm{~nm}}$
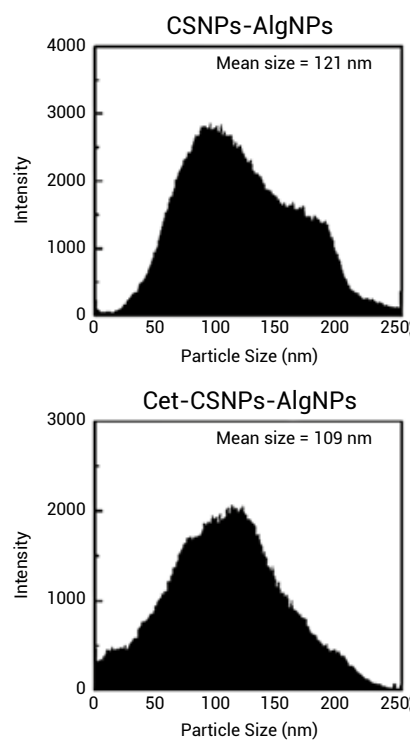
Table 1. Zeta potential of CSNPs, Cet-CSNPs, AlgNPs, Cet-AlgNPs, CSNPs-AlgNPs and Cet-CSNPs-AlgNPs.

\begin{tabular}{|c|c|}
\hline Sample & Zeta potential $/ \mathrm{mV}$ \\
\hline CSNPs & $+15.0 \pm 2.5$ \\
\hline Cet-CSNPs & $+6.7 \pm 4.2$ \\
\hline AlgNPs & $-4.2 \pm 1.1$ \\
\hline Cet-AlgNPs & $-5.6 \pm 2.0$ \\
\hline CSNPs-AlgNPs & $+8.2 \pm 4.3$ \\
\hline Cet-CSNPs-AlgNPs & $-6.4 \pm 3.3$ \\
\hline
\end{tabular}

\section{The effect of $\mathrm{pH}$ on the Zeta potential of the nanoparticles}

Particle charge is one of the stability-determining factors and their Zeta potential value is usually between $>+30 \mathrm{mV}$ or $<-30 \mathrm{mV}$. This is the ideal value for the physical stability of any nanoparticles (36). The zeta potential of CSNPs and Cet-CSNPs was determined in this work and it was decreased from $+15.0 \mathrm{mV}$ in CSNPs to $+6.7 \mathrm{mV}$ in Cet-CSNPs as shown in Table 1. During the synthesis of CSNPs, the CS and TPP were mixed into acid media solutions, such as acetic acid. They formed solid nanoparticles with a positive surface charge, and the density of the surface charge is measured by the zeta potential values (36).

The zeta potential for AlgNPs and Cet-AlgNPs was found to be $-4.2 \mathrm{mV}$ and $-5.6 \mathrm{mV}$, respectively as shown in Table 1. The surface charges of AlgNPs and Cet-AlgNPs were negative, due to the electric potential of the nanoparticles. It was influenced by the composition of the starting materials and the medium they dispersed into (37). This result was in agreement with the previous study (38). The AlgNPs and Cet-AlgNPs with a small value of zeta potential $(-4.2 \mathrm{mV}$ and $-5.6 \mathrm{mV})$ are due to higher $\mathrm{Alg}$ content and the amounts of $\mathrm{CaCl}_{2}$, and Cet was

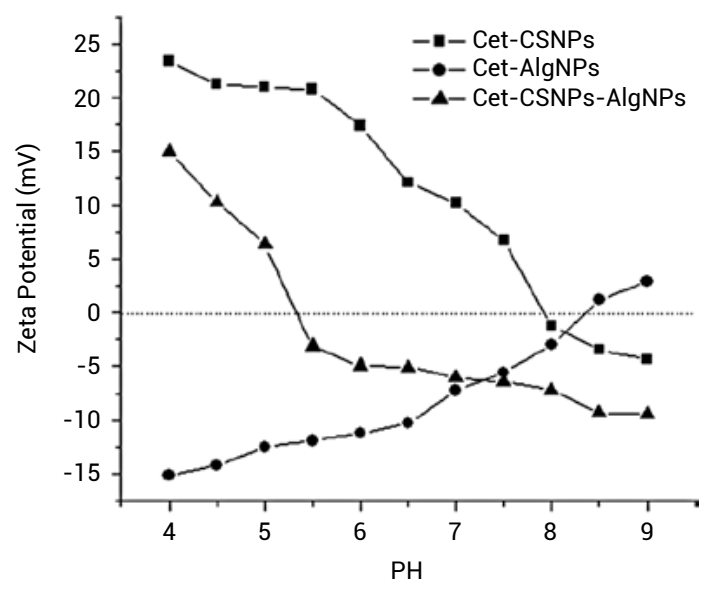

Figure 5. The effect of $\mathrm{pH}$ on the zeta potential of the nanoparticles. still insufficient to interact with all the carboxylic groups of Alg (39).

Zeta potential of CSNPs-AlgNPs and CetCSNPs-AlgNPs was determined and it was changed from $+8.2 \mathrm{mV}$ for CSNPs-AlgNPs to $-6.4 \mathrm{mV}$ for Cet-CSNPs-AlgNPs as shown in Table 1. The positive charge for CSNPs-AlgNPs was dependent on the total protonated $\mathrm{NH}_{3}^{+}$groups of CS and their neutralization with carboxylic groups of $\mathrm{Alg}$, i.e. the $\mathrm{Alg} / \mathrm{CS}$ ratio. When the amount of CS exceeded the amount of Alg, the nanoparticles showed positively charged zeta potential, which indicated that the $\mathrm{NH}_{3}^{+}$groups of CS were sufficiently neutralized by the carboxylic groups of Alg. The Cet-CSNPs-AlgNPs have a negative charge which is due to the presence of Cet with carboxylate anion.

Figure 5 shows the change in the zeta potential of three nanoparticles from acidic $(\mathrm{pH} 4)$ to alkaline ( $\mathrm{pH}$ 8.5). For Cet-CSNPs, when the $\mathrm{pH}$ was increased by the addition of $\mathrm{NaOH}$ solution, the zeta potential was decreased from $+23.4 \mathrm{mV}$ to $-4.4 \mathrm{mV}$. In the case of Cet-AlgNPs, the potential was changed from $-15.1 \mathrm{mV}$ to $-3.9 \mathrm{mV}$, whereas for Cet-CSNPs-AlgNPs, the potential also was changed from $+15.3 \mathrm{mV}$ to $-9.4 \mathrm{mV}$. The isoelectric point ( $\mathrm{pI}$ ) can be defined as the $\mathrm{pH}$ in which the net charge of the particle is zero. It is the point where the positive charges are completely neutralized by the negative charges. The pI value for Cet-CSNPs, Cet-AlgNPs, and Cet-CSNPs-AlgNPs are 7.9, 8.3, and 5.4 , respectively.

\section{Thermogravimetric analysis}

Thermogravimetric analyses (TGA) were obtained for Cet, CSNPs, Cet-CSNPs, AlgNPs, CetAlgNPs, CSNPs-AlgNPs, and Cet-CSNPs-AlgNPs and are shown in Figure 6. For Cet, the TGA thermogram shows a single sharp weight loss starting at $160^{\circ} \mathrm{C}$ with a maximum temperature of $301^{\circ} \mathrm{C}$ and a total weight loss of $91.2 \%$. Therefore Cet was found to be stable up to $160^{\circ} \mathrm{C}(40)$. The TGA curve of the CSNPs is shown in Figure 6A. The initial weight loss at $60^{\circ} \mathrm{C}$ is due to the evaporation of $\mathrm{H}_{2} \mathrm{O}$, while the second weight loss between $200-400^{\circ} \mathrm{C}$ is due to decomposition and the dehydration of the saccharide structure, depolymerization, and finally, decomposition of the acetylated and deacetylated polymer, with a peak observed at $228^{\circ} \mathrm{C}$. The TGA curve of Cet-CSNPs in Figure 6A shows the same weight loss steps for CSNPs. The difference between them lies in the total weight loss, as the total weight of the CetCSNPs is higher than the CSNPs.

TGA analysis in Figure 6B gives some information about the thermal behavior of the AlgNPs. 


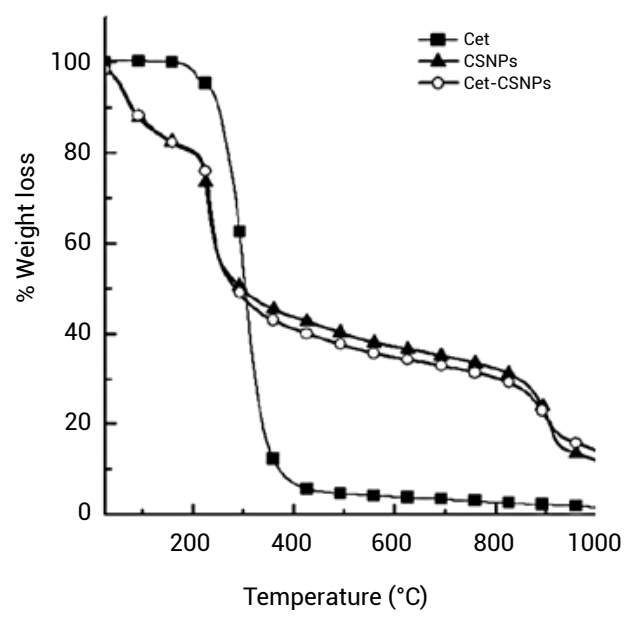

B

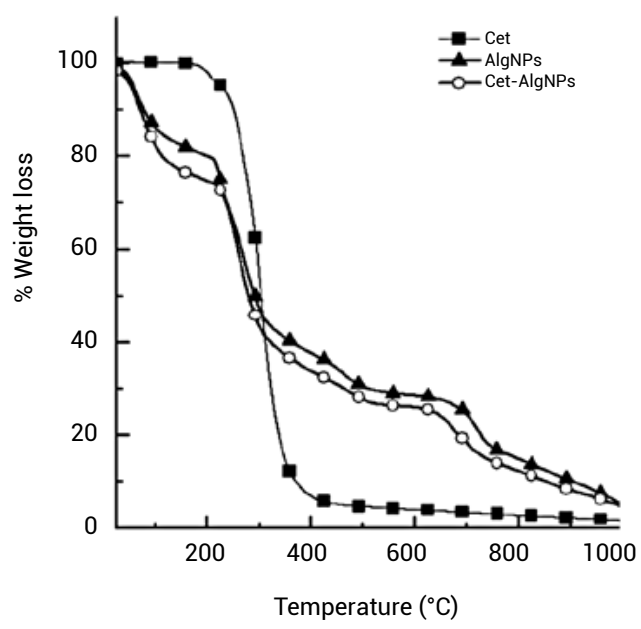

C

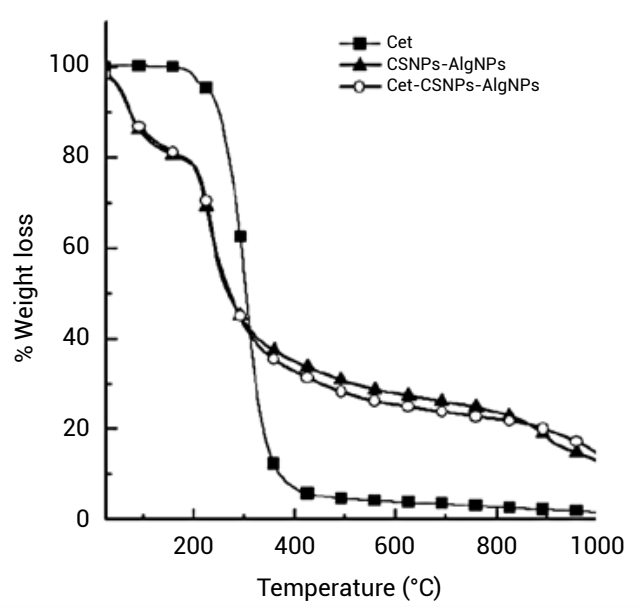

Figure 6.TGA thermograms of Cet, CSNPs, Cet-CSNPs, AlgNPs, Cet-AlgNPs, CSNPs-AlgNPs and Cet-CSNPs-AlgNPs nanoparticles.

The first weight loss between $100-300^{\circ} \mathrm{C}$ is due to the loss of bound (residual) and unbound (moisture) water molecules from the polymer matrix, as well as the decomposition of the saccharide structure. The second stage of weight loss observed between $300-800^{\circ} \mathrm{C}$ could be related to the removal of saccharide molecules from the polymer structure and the complete degradation and decomposition of the polymer main chain. The TGA thermogram of CetAlgNPs shown in Figure 6B shows the same thermal behavior as that of AlgNPs. The second stage of weight loss in the temperature range of $300-800^{\circ} \mathrm{C}$ is due to the degradation of Cet as well as AlgNPs with $53 \%$ weight loss.

The decomposition of Cet-CSNPs-AlgNPs shows two major and one minor weight loss (Figure 6C). The thermograms of Cet, CSNPs, and AlgNPs are shown in Figure 4E. The first weight loss at $62^{\circ} \mathrm{C}$ is due to moisture that is present from the polymer structure with $14.3 \%$ weight loss. However, when the temperature was reached at $227^{\circ} \mathrm{C}, 42.2 \%$ weight loss was observed, which is due to the decomposition of Cet and the complexes of CSNPs-AlgNPs. The second major step of weight loss occurred at $750-800^{\circ} \mathrm{C}$, which is due to CSNPs.

\section{In vitro study of Cet release from its nanoparticles}

The release behaviors for Cet from Cet-CSNPs, Cet-AlgNPs, and Cet-CSNPs-AlgNPs, and the physical mixture of Cet with CSNPs, AlgNPs, and CSNPs-AlgNPs in a phosphate-buffered solution at $\mathrm{pH} 7.4$ are shown in Figure 7. It can be seen that the physical mixture, when exposed to the phosphate-buffered solution at 7.4, released Cet quickly and reached $90 \%, 95 \%$, and $86 \%$ at $25 \mathrm{~min}$ for CSNPs, AlgNPs, and CSNPs-AlgNPs, respectively. The release behaviors of Cet from nanoparticles were very much lower than that from the physical mixture, indicating that the nanoparticles are 

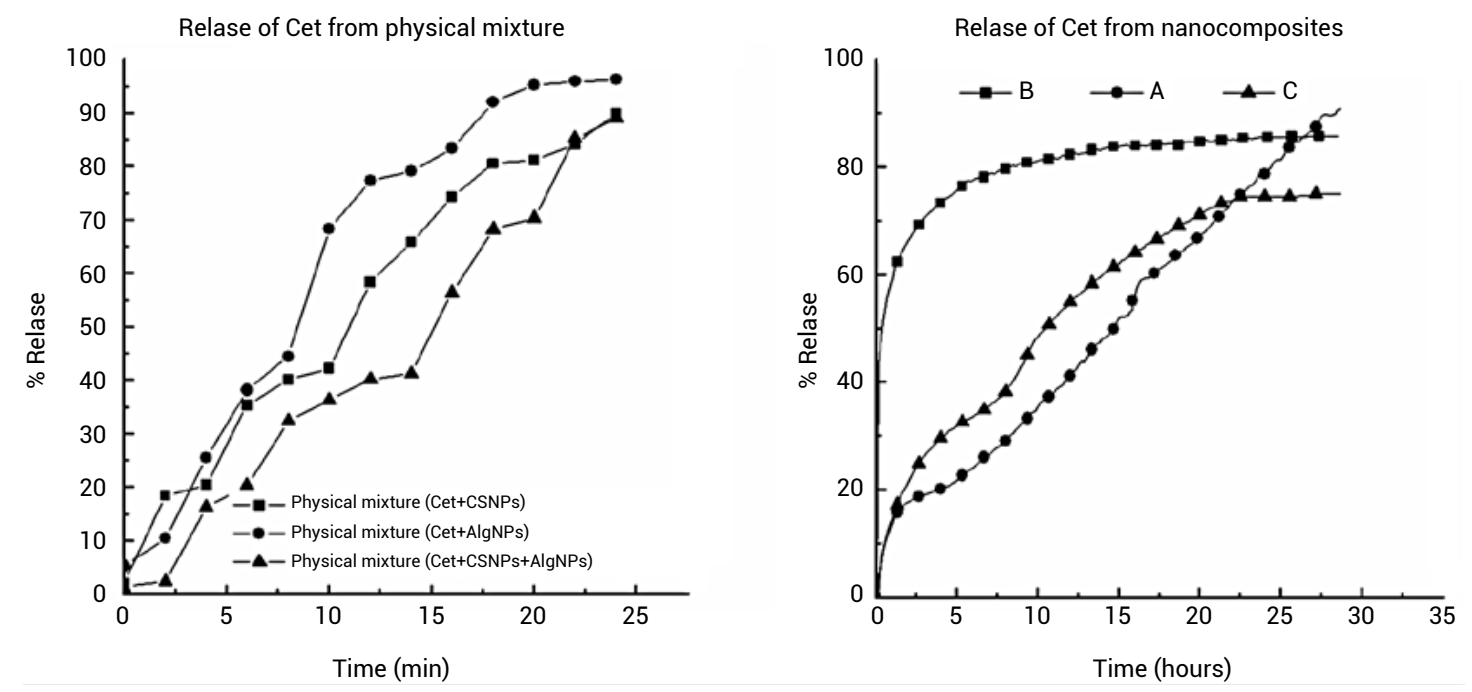

Figure 7. The release profiles of Cet from its Cet-CSNPs (A), Cet-AlgNPs (B), and Cet-CSNPs-AlgNPs (C) into PBS solution at pH 7.4 and from its physical mixture.

a potential controlled-release system. This may be due to the electrostatic attraction between the Cet and the nanoparticles.

Drugs can be released from their polymer hosts by three mechanisms; swelling-diffusion (41), diffusion and erosion, or degradation of polymers (42). The swelling mechanism occurs for hydrophilicity polymer and it depends on the $\mathrm{pH}$ (43). The polymer nanoparticles are initially dry, and when they are added into the release media, they swell to increase their porosity, leading to the diffusion of the drug molecules (44). In the diffusion mechanism, the drug moves from the inner polymeric matrix to the release medium. Polymer chains form the diffusion barrier, and this barrier will make the release of the drug difficult and therefore drug is released in a controlled manner.

Erosion and degradation of polymers take place when the polymer chains convert to lower molecular weight, effectively releasing drug molecules that were trapped by the polymer chains. The erosion mechanism occurs in two different ways: homogenous and heterogeneous. In homogenous erosion, the polymer is eroded at the same rate throughout the matrix. On the other hand, in heterogeneous erosion, polymer erosion takes place from the surface towards the inner core. Polymer degradation may be caused due to the $\mathrm{pH}$ surrounding the media or the presence of enzymes (45).

Release studies of Cet from Cet-CSNPs, CetAlgNPs, and Cet-CSNPs-AlgNPs were carried out using PBS medium at $\mathrm{pH}$ 7.4. Figure 7B shows that about $70 \%$ of the drug is fast released in the first $2 \mathrm{~h}$. This result indicates that part of the drug is localized as a free drug on the surface of the nanoparticles. In addition, AlgNPs are unstable at pH 7.4 (46), and the release of the drug follows the swelling mechanism (47). After this initial release, the drug release is almost constant, and around $85 \%$ of the Cet was released from the Cet-AlgNPs in $27 \mathrm{~h}$.

Figures 7A and $\mathrm{C}$ show that both nanoparticles, Cet-CSNPs and Cet-CSNPs-AlgNPs, are able to release about $90 \%$ and $75 \%$ of the Cet, respectively, in $30 \mathrm{~h}$. Also in both nanoparticles, the release of the Cet drug was found to follow the diffusion through the polymers matrix. This is because CSNPs is $\mathrm{pH}-$ dependent, it is faster at a lower $\mathrm{pH}$ than around neutral $\mathrm{pH}(\mathrm{pH} 5.2>\mathrm{pH} 6.8>\mathrm{pH}$ 7.4) (48).

The mean dissolution time value is used to describe the drug release rate from the polymer, and the efficiency of the polymer. A higher value of MDT indicates a slower release rate (49). The MDT value for Cet-CSNPs, Cet-AlgNPs, and Cet-CSNPs-AlgNPs was found to be $10.5,37.4$, and $22.5 \mathrm{~h}$, respectively. These results reflect modification of the release performance from rapid for Cet-AlgNPs to sustained release for Cet-CSNPs, and Cet-CSNPs-AlgNPs.

\section{Release kinetics of Cet from its nanoparticles}

The data of the cumulative release of Cet from its nanoparticles was fitted to four kinetic models which are generally described in Table 2 (50).

Table 3 and Figure 8 show that the release of Cet from Cet-CSNPs was best fitted to the HixsonCrowell model with $\mathrm{R}^{2}=0.9631$. On the other hand, for Cet-AlgNPs, Cet release was best fitted to the pseudo-second-order, with $\mathrm{R}^{2}=0.9998$. In addition, the release of Cet from Cet-CSNPs-AlgNPs 
Table 2. Kinetic models that were used in the fitting of the cumulative release of Cet from its nanoparticles (Dong et al., 2010, Ho and Ofomaja, 2006, Sakore and Chakraborty, 2013).

\begin{tabular}{|c|c|}
\hline Kinetic model & Equation \\
\hline First-order & $\ln \left(\mathrm{q}_{\mathrm{e}}-\mathrm{q}_{\mathrm{t}}\right)=\ln \mathrm{q}_{\mathrm{e}}-\mathrm{k}_{1} \mathrm{t}$ \\
\hline Second-order & $\mathrm{t} / \mathrm{q}_{\mathrm{t}}=1 / \mathrm{k}_{2} \mathrm{q}_{\mathrm{e}}^{2}+\mathrm{t} / \mathrm{q}_{\mathrm{e}}$ \\
\hline Higuchi & $\mathrm{q}_{\mathrm{t}}=\mathrm{K}_{\mathrm{H}} \sqrt{\mathrm{t}}$ \\
\hline Hixson-Crowell & $\sqrt[3]{\mathrm{M}_{\mathrm{o}}}-\sqrt[3]{\mathrm{q}_{\mathrm{t}}}=\mathrm{Kt}$ \\
\hline
\end{tabular}

$\mathrm{q}_{\mathrm{e}}$ is the quantity released at equilibrium, $\mathrm{q}_{\mathrm{t}}$ is the quantity released at any time $\mathrm{t}, \mathrm{k}_{\mathrm{n}}$ is the rate constant of the $\mathrm{n}$-order release kinetics, and $\mathrm{M}_{\mathrm{o}}$ is the initial quantity of drug in the nanoparticles

Table 3. The correlation coefficients $\left(\mathrm{R}^{2}\right)$ and constant rate model obtained by fitting the Cet release data from the Cet-CSNPs, Cet-AlgNPs, Cet-CSNPs-AlgNPs in PBS solutions at $\mathrm{pH}$ 7.4.

\begin{tabular}{|c|c|c|c|c|c|c|c|c|c|}
\hline \multirow{2}{*}{ Samples } & \multirow{2}{*}{$\begin{array}{c}\text { Release } \\
(\%)\end{array}$} & \multicolumn{2}{|c|}{$\begin{array}{c}\text { Pseudo-first } \\
\text { order }\end{array}$} & \multicolumn{2}{c|}{$\begin{array}{c}\text { Pseudo-second } \\
\text { order }\end{array}$} & \multicolumn{3}{c|}{$\begin{array}{c}\text { Higuchi } \\
\text { model }\end{array}$} & $\begin{array}{c}\text { Hixson-Crowell } \\
\text { model }\end{array}$ \\
\cline { 3 - 10 } & & $\mathrm{R}^{2}$ & $\mathrm{~K}_{\mathrm{o}}$ & $\mathrm{R}^{2}$ & $\mathrm{~K}_{2}$ & $\mathrm{R}^{2}$ & $\mathrm{~K}_{\mathrm{H}}$ & $\mathrm{R}^{2}$ & $\mathrm{~K}_{\mathrm{HC}}$ \\
\hline Cet-CSNPs & 90 & 0.921 & $7.0 \times 10^{-2}$ & 0.593 & $2.1 \times 10^{-4}$ & 0.943 & $5.0 \times 10^{-2}$ & 0.963 & $7.9 \times 10^{-2}$ \\
\hline Cet-AlgNPs & 85 & 0.736 & $3.5 \times 10^{-2}$ & 0.999 & $1.5 \times 10^{-2}$ & 0.661 & $1.0 \times 10^{-1}$ & 0.670 & $3.3 \times 10^{-2}$ \\
\hline Cet-CSNPs-AlgNPs & 75 & 0.972 & $5.1 \times 10^{-2}$ & 0.946 & $8.0 \times 10^{-4}$ & 0.980 & $6.2 \times 10^{-2}$ & 0.960 & $6.0 \times 10^{-2}$ \\
\hline
\end{tabular}
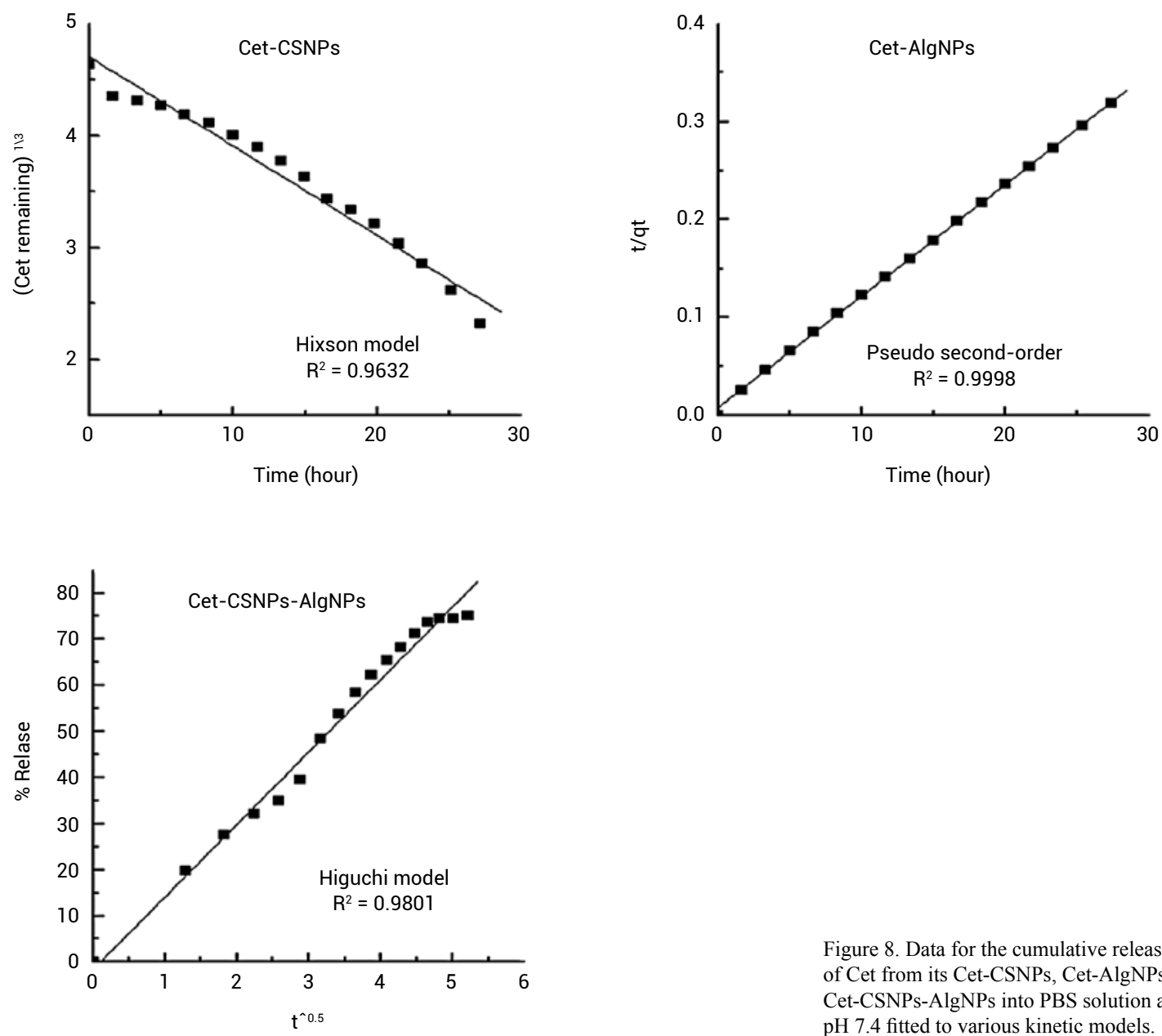

Figure 8. Data for the cumulative release of Cet from its Cet-CSNPs, Cet-AlgNPs, Cet-CSNPs-AlgNPs into PBS solution at $\mathrm{pH} 7.4$ fitted to various kinetic models. 


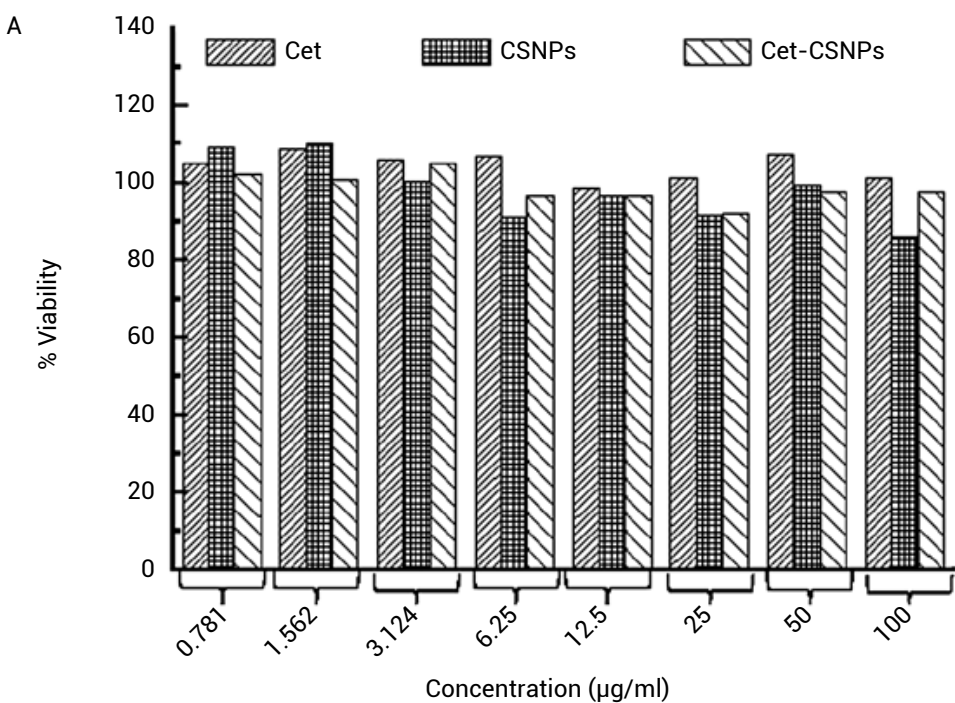

B
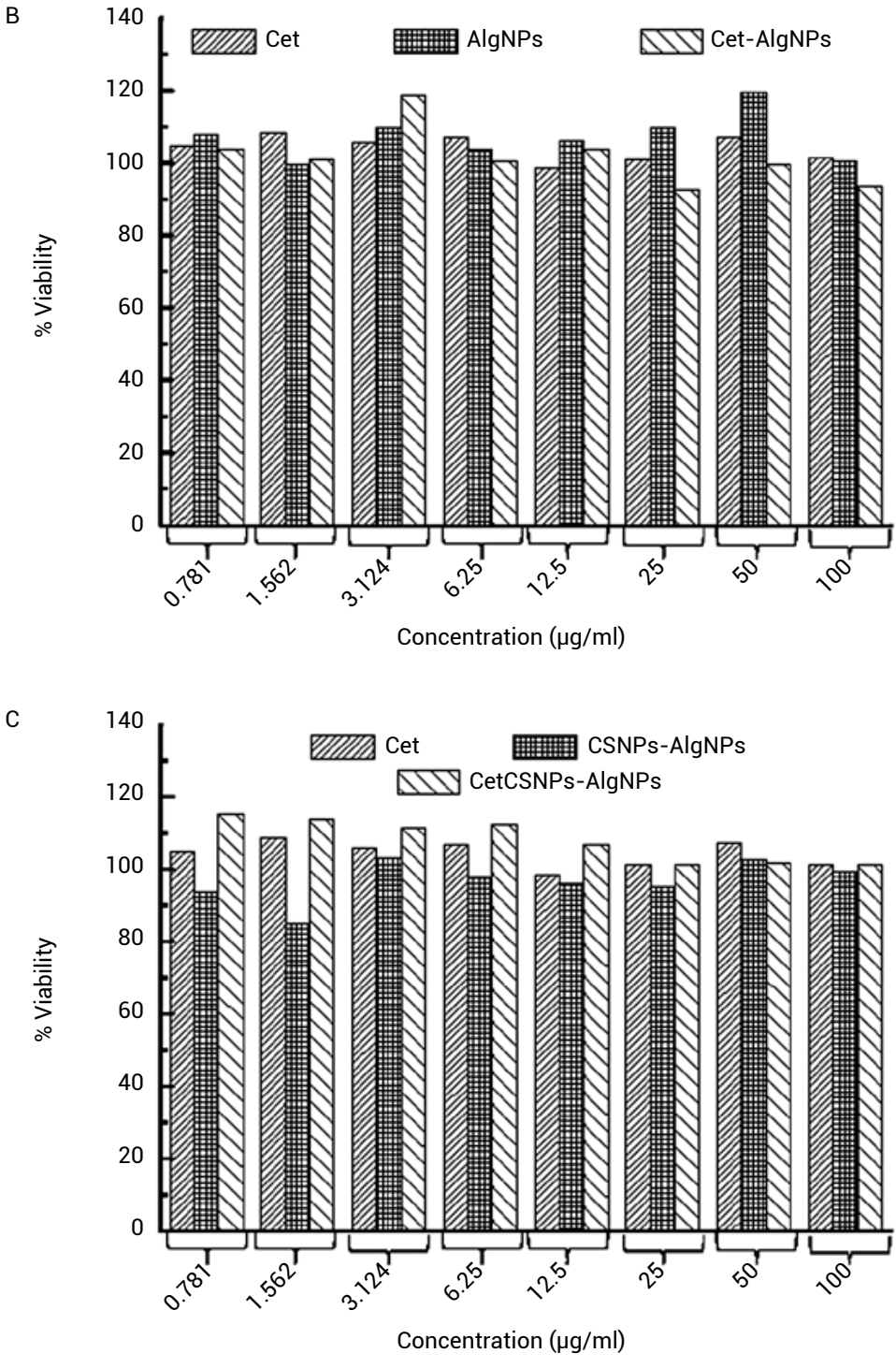

was found to follow the Higuchi model with $\mathrm{R}^{2}=0.9802$.

\section{Cytotoxicity studies}

In this study, the potential cytotoxicity of nanoparticles on 3T3 cells was evaluated by the MTT assay. The results are shown in Figures 9A-C. In vitro, MTT results showed that blank CSNPs, AlgNPs, and CSNPs-AlgNPs, as well as the nanoparticles of Cet-CSNPs, Cet-AlgNPs, and Cet-CSNPsAlgNPs, show no significant cytotoxicity after $24 \mathrm{~h}$. These results were consistent with the previous reports that CS and Alg are natural carriers with low toxicity and biodegradability (51).

\section{CONCLUSION}

The findings of this study show that various nanoparticles, Cet-CSNPs, Cet-AlgNPs, and Cet-CSNPs-AlgNPs, can be prepared by the ionic gelation method. The resulting nanoparticles are generally of amorphous phase with a mean size of 96,95 , and $109 \mathrm{~nm}$, respectively, where there exists an interaction between Cet with CSNPs, AlgNPs, and CSNPs-AlgNPs. Cet was found to be less loaded on CSNPs than AlgNPs; 20 and 45\%, respectively. The combination of CSNPs and AlgNPs i.e. CSNPs-AlgNPs gave almost the average value of the two polymers, i.e. $35 \%$. However, there is no clear trend between the size of the nanoparticles with the polymer that can be observed. Similarly, there is no specific relationship between the release of Cet from its nanoparticles to the kinetic release mechanism; they follow the HixsonCrowell, Pseudo-second, and

Figure 9. The cytotoxicity profiles for the Cet, Cet-CSNPs, Cet-AlgNPs, and Cet-CSNPs-AlgNPs toward $3 \mathrm{~T} 3$ cells. 
Higuchi models for Cet-CSNPs, Cet-AlgNPs, and Cet-CSNPs-AlgNPs, respectively. However, these preliminary results are useful to be used as the starting points for further in vivo studies.

\section{Funding acknowledgment}

The author would like to thank the project supported by the Faculty of Pharmacy, Isra University, and the Ministry of Higher Education of Malaysia (MOHE) under grant No. UPM/800-3/3/1/ GPB/2019/9678800.

\section{Conflict of interest}

The authors declare no conflicts of interest.

\section{REFERENCES}

1. Khan G., Yadav S.K., Patel R.R., Nath G., Bansal M., Mishra B.: AAPS PharmSciTech 17, 1312 (2016).

2. Lu Y., Zhang E., Yang J., Cao Z..: Nano Res. 11, 4985, (2018).

3. Endo K., Ueno T., Kondo S., Wakisaka N., Murono S., et al.: Cancer Sci. 104, 369 (2013).

4. Han J., Zhao D., Li D., Wang X., Jin Z., Zhao K.: Polymers (Basel) 10, 31 (2018).

5. Sabbagh H.A.K., Hussein-Al-Ali S.H., Hussein M.Z., Abudayeh Z., Ayoub R., Abudoleh S.M.: Polymers (Basel) 12, 772 (2020).

6. Bedade D.K., Sutar Y.B., Singhal R.S.: Food Chem. 275, 95 (2019).

7. Gomes J., Belinha J., Jorge R.N., Eds.: Application of chitosan in dentistry - a review. Biodental Engineering V: Proceedings of the 5 th International Conference on Biodental Engineering, Porto, Portugal 2019.

8. Abdollahimajd F., Moravvej H., Dadkhahfar S., Mahdavi H., Mohebali M., Mirzadeh H.: Indian J. Dermatol. Venereol. Leprol. 85, 609 (2019).

9. Kenawy E., Omer A.M., Tamer T.M., Elmeligy M.A., Eldin M.S.M.: Int. J. Biol. Macromol. 139, 440 (2019).

10. Newton A., Prabakaran L.: Comparative efficacy of chitosan, pectin based Mesalamine colon targeted drug delivery systems on TNBSinduced IBD model rats. Anti-inflammatory \& anti-allergy agents in medicinal chemistry (2019).

11. Shariatinia Z., Mazloom-Jalali A.: J. Mol. Liq. 273, 346 (2019).

12. Sydow S., Aniol A., Hadler C., Menzel H: Biomolecules 9, 573 (2019).
13. Smitha B., Sridhar S., Khan A.A.: Eur. Polym. J. 41, 1859 (2005).

14. Thünemann A.F., Müller M., Dautzenberg H., Joanny JF., Löwen H.: Adw. Polym. Sci. 166, 113 (2004).

15. Slater J.W., Zechnich A.D., Haxby D.G.: Drugs 57, 31 (1999).

16. Hasan S., Al Ali H., Al-Qubaisi M., Zobir Hussein M., Ismail M., et al.: Int. J. Nanomedicine 7, 3351 (2012).

17. Gholivand M.B., Shamsipur M., Ehzari H.: Microchem. J. 146, 692 (2019).

18. Goindi S., Kumar G., Kaur A.: J. Liposome Res. 24, 249 (2014).

19. Kirimlioğlu G.Y., Öztürk A.A.: Turk. J. Pharm. Sci. 17, 27 (2020).

20. Li F.Q., Ji R.R., Chen X., You B.M., Pan Y.H., Su J.C.: Arch. Pharm. Res. 33, 1967 (2010).

21. Yu X., Mu Y., Xu M., Xia G., Wang J., et al.: Carbohydr. Polym. 173, 600 (2017).

22. Sambale F., Wagner S., Stahl F., Khaydarov R.R., Scheper T., et al.: J. Nanomater. 2015, 9 pages (2015).

23. Mosmann T.: J. Immunol. Methods 65, 55 (1983).

24. Hasan S., Al Ali H., Al-Qubaisi M., Zobir Hussein M., Ismail M., et al.: Int. J. Nanomedicine 7, 3351 (2012).

25. Nagarwal RC, Singh PN, Kant S, Maiti P, Pandit JK.: Chem. Pharm. Bull. (Tokyo) 59, 272 (2011).

26. Zhou H., Yu W., Guo X., Liu X., Li N., et al.: Biomacromolecules 11, 3480 (2010).

27. Fang D., Liu Y., Jiang S., Nie J., Ma G.: Carbohydr. Polym. 85, 276 (2011).

28. Paczkowska M., Mizera M., Lewandowska K., Kozak M., Miklaszewski A., et al.: J. Incl. Phenom. Macrocycl. Chem. 91, 149 (2018).

29. de Souza Costa-Júnior E., Pereira M.M., Mansur H.S.: J. Mater. Sci. Mater. Med. 20, 553 (2009).

30. Daemi H., Barikani M.: Sci. Iran. 19, 2023 (2012).

31. Kulig D., Zimoch-Korzycka A., Jarmoluk A., Marycz K.: Polymers (Basel) 8, 167 (2016).

32. Wu J., Wang Y., Yang H., Liu X., Lu Z.: Carbohyd. Polym. 175, 170 (2017).

33. Hussein-Al-Ali S.H., Kura A., Hussein M.Z., Fakurazi S.: Polym. Compos. 39, 544 (2018).

34. Biswal T., Barik B., Sahoo P.K.: J. Mater. Sci. Nanotechnol. 4, 203 (2016).

35. Li P., Dai Y.N., Zhang J.P., Wang A.Q., Wei Q.: Int. J. Biomed. Sci. 4, 221 (2008).

36. Nallamuthu I., Devi A., Khanum F.: Asian J. Pharm. Sci. 10, 203 (2015). 
37. Sumathi R., Tamizharasi S., Sivakumar T.: Int. J. App. Pharm. 9, 60 (2017).

38. Morsi N., Ghorab D., Refai H., Teba H.: Int. J. Pharm. Pharm. Sci. 7, 234 (2015).

39. Ahdyani R., Novitasari L., Martien R., Danarti R.: Int. J. App. Pharm. 11, 48 (2019).

40. Hussein Al Ali S.H., Al-Qubaisi M., Hussein M.Z., Zainal Z., Hakim M.N.: Int. J. Nanomedicine 6, 3099 (2011).

41. Liu S., Yang S., Ho P.C.: Asian J. Pharm. Sci. 13, 72 (2018).

42. Singh R., Lillard J.W. Jr.: Exp. Mol. Pathol. 86, 215 (2009).

43. Fonseca-Santos B., Chorilli M.: Mater. Sci. Eng. C Mater. Biol. Appl. 77, 1349 (2017).
44. Bae K.H., Chung H.J., Park T.G.: Mol. Cells 31, 295 (2011).

45. Göpferich A.: Biomaterials 17, 103 (1996).

46. Sambu S., Xu X., Ye H., Cui Z.F.: Proc. Inst. Mech. Eng. H 225, 1092 (2011).

47. Bajpai S.K., Sharma S.: React. Funct. Polym. 59, 129 (2004).

48. Bhavin K.P., Rajesh H.P., Pooja S.A.: J. Drug Delivery 2013, 10 pages (2013).

49. Rinaki E., Dokoumetzidis A., Macheras P.: Pharm. Res. 20, 406 (2003).

50. Gouda R., Baishya H., Qing Z.J.J.D.D.: J. Dev. Drugs 6, 171 (2017).

51. Kumar S.P., Birundha K., Kaveri K., Devi K.T.: Int. J. Biol. Macromol. 78, 87 (2015). 